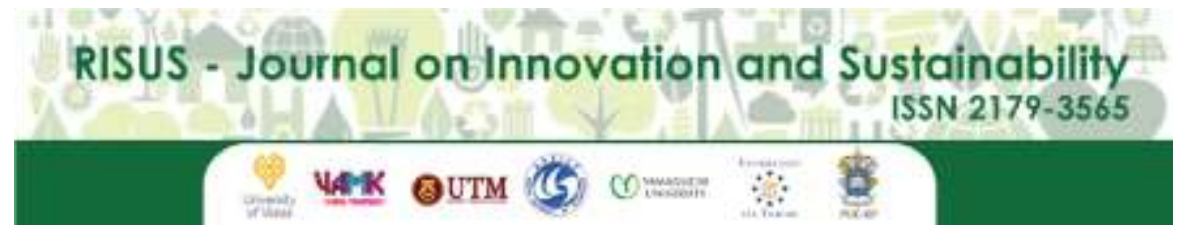

RISUS - Journal on Innovation and Sustainability volume 10, número 3 - 2019

ISSN: $2179-3565$

Editor Científico: Arnoldo José de Hoyos Guevara Editor Assistente: Luciano da Silva Ferreira Leite Avaliação: Melhores práticas editoriais da ANPAD

\title{
NANOFLUIDS: LOOKING FOR MORE EFFICIENCY IN SOLAR HEATING SYSTEMS
}

Nanofluídos: Buscando maior eficiência em sistemas de aquecimento solar

Etevaldo Francsico Carreira Júnior; etevaldo.carreira@ufabc.edu.br; https://orcid.org/00000003-1012-4878; Universidade Federal do ABC

Reynaldo Palacios Berecher; reynaldo.palacios@ufabc.edu.br;

UniversidadeFederal do ABC

Paulo Henrique de Mello Santana; paulo.santana@ufabc.edu.br

Universidade Federal do ABC

\begin{abstract}
Researches into the use of solar energy and its application in the form of heat have been intensified in the recent history of renewable energy. However, flat plate and evacuated tube solar collectors still have low capacity to convert solar energy to thermal energy due to several factors, including low thermal conductivity of the exchange fluid. Recent studies seek to make feasible the use of nanofluids, whose thermophysical properties, such as thermal conductivity, can improve the performance of solar collectors. The objective of this paper is to review the recent literature on solar water heating systems, identifying opportunities to apply nanofluids to solar collectors, in order to increase their efficiency. The results of the research point to gains of more than $20 \%$ in the efficiency of the collectors compared to the use of water as thermal exchange fluid, and also indicate that the carbon nanoparticles have the best cost-benefit ratio, although the nanofluids are not yet feasible for real applications, because there are challenges to be overcome by this technology, such as reducing the loss of stability, which reduces its useful life, reduce the complexity and cost of production for commercial scale production. It is concluded that there is in fact potential to apply nanofluids in solar thermal systems.
\end{abstract}

KEY WORDS: Solar energy. Solar heating, Water heating, Nanofluids, Alternative energy source

\author{
ACEITO EM: 15/08/2019
}




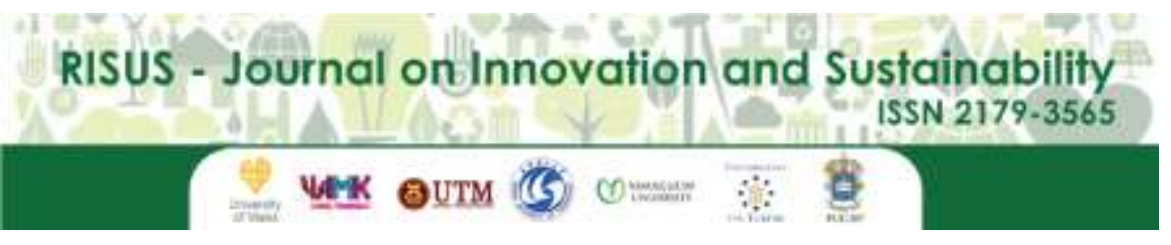

RISUS - Journal on Innovation and Sustainability volume 10, número 3 - 2019 ISSN: $2179-3565$

Editor Científico: Arnoldo José de Hoyos Guevara Editor Assistente: Luciano da Silva Ferreira Leite Avaliação: Melhores práticas editoriais da ANPAD

\title{
NANOFLUÍDOS: BUSCANDO MAIOR EFICIÊNCIA EM SISTEMAS DE AQUECIMENTO SOLAR \\ Nanofluids: looking for more efficiency in solar heating systems
}

Etevaldo Francsico Carreira Júnior; etevaldo.carreira@ufabc.edu.br; https://orcid.org/00000003-1012-4878; Universidade Federal do ABC

Reynaldo Palacios Berecher; reynaldo.palacios@ufabc.edu.br; UniversidadeFederal do ABC

Paulo Henrique de Mello Santana; paulo.santana@ufabc.edu.br Universidade Federal do ABC

\begin{abstract}
RESUMO: Pesquisas para a utilização da energia solar e seu aproveitamento na forma de calor têm sido intensificadas na história recente das energias renováveis. Entretanto, coletores solares de placa plana e de tubo evacuado ainda apresentam baixa capacidade de converter energia solar em energia térmica devido a vários fatores, entre eles a baixa condutividade térmica do fluído de troca. Estudos recentes buscam viabilizar o uso de nanofluídos, cujas propriedades termofísicas, como a condutividade térmica, podem melhorar o desempenho dos coletores solares. O objetivo deste artigo é revisar a literatura recente sobre sistemas de aquecimento solar de água, identificando oportunidades de aplicar nanofluídos em coletores solares, visando aumentar a eficiência dos mesmos. Os resultados das pesquisas apontam para ganhos acima de $20 \%$ na eficiência dos coletores, em comparação à utilização de água como fluído de troca térmica, e indicam também que as nanopartículas de carbono apresentam a melhor relação custo-benefício, embora os nanofluídos ainda não sejam viáveis para aplicações reais, pois há desafios a serem superados por essa tecnologia como, por exemplo, reduzir a perda de estabilidade, o que diminui sua vida útil, reduzir a complexidade e o custo de obtenção para produção em escala comercial. Conclui-se que há de fato potencial para aplicar nanofluídos em sistemas termossolares.
\end{abstract}

Palavras-chave: Energia solar, Aquecimento solar, Aquecimento de água, Nanofluídos, Fontes alternativas de energia.

\section{ACEITO EM: 15/08/2019}




\section{INTRODUÇÃO}

Com o aumento das demandas de energia em todo o mundo, há uma intensificação das pesquisas para a utilização de fontes de energia renovável. Dentre as alternativas existentes, a energia solar pode ser considerada como a mais extensivamente disponível, que converte diretamente a energia solar em eletricidade e calor, sem qualquer emissão de gases de efeito estufa. Os coletores mais comuns são os de placa plana, mais baratos e convenientes para temperaturas até $80^{\circ} \mathrm{C}$, fáceis de utilizar e necessitam pouca manutenção. Coletores de tubos evacuados também são bastante comuns e podem atingir temperaturas superiores, até $130^{\circ} \mathrm{C}$, são construídos de tubos de vidro a vácuo, mais complexos e mais caros que os de placa plana. Em relação ao aproveitamento da energia solar na forma de calor, Sarsam et al. (2015) refere-se aos coletores solares de placa plana como um dos tipos de coletores solares mais amplamente utilizados e conhecidos, mas cuja eficiência é limitada tanto pela placa de absorção do coletor para absorver a energia solar, quanto pela transferência da energia solar para o fluído no coletor. Raj e Subudhi (2018) também se referem ao coletor solar de placa plana como sendo o coletor solar mais usado e menos eficiente, destacando em seu estudo que o uso de um nanofluído no lugar do fluído base resulta em melhor desempenho do coletor solar.

Kasaeian et al. (2015) enfatizam que a nanotecnologia moderna pode produzir partículas metálicas ou não metálicas de dimensões nanométricas que possuem propriedades mecânicas, ópticas, elétricas, magnéticas e térmicas exclusivas e que sua aplicação em sistemas solares oferece vantagens únicas em relação aos fluídos convencionais, não somente em coletores planos, mas também nos coletores solares de tubos evacuados. Verma e Tiwari (2015) salientam que a energia solar tem atraído muito a atenção dos cientistas, interessados principalmente na inovação em novos dispositivos, bem como em desenvolver novos métodos para aproveitá-la, tendo como principais focos a miniaturização de dispositivos e o aumento da eficiência energética por meio dos novos materiais, dentre os quais os nanofluídos, que representam uma inovação comparativamente recente que exibe melhor capacidade de absorção e de transporte de calor.

Sistemas energéticos de transferência de calor dependem de fluídos convencionais como a água, o etilenoglicol ou óleos, cujas propriedades termofísicas limitam o desenvolvimento desses sistemas, bem como a sua miniaturização.

Estudos sobre a transferência de calor por nanofluídos permitiram identificar relações entre as diferentes variáveis como propriedades termo físicas, fração volumétrica, temperatura e velocidade de escoamento, encontrando-se taxas superiores de transferência de calor por convecção, quando comparadas ao fluído base. A aplicabilidade dos nanofluídos na indústria solar térmica tem despertado bastante interesse, devido à capacidade dos nanofluídos em absorver a radiação solar. A otimização dos equipamentos de troca de calor depende de três fatores: área de transferência de calor, gradiente de temperaturas e coeficiente de transferência de calor do fluído operante. $\mathrm{O}$ aumento da área de transferência de calor é uma estratégia comum para melhorar a taxa de transferência de calor do sistema. Contudo, em situações de restrições ao tamanho e ao peso este tipo de otimização não é viável. A alteração do gradiente de temperaturas permite tornar a transferência de calor mais eficiente, no entanto, isso torna-se uma desvantagem para os coletores solares, pois requer maior intensidade de energia solar para o funcionamento do equipamento, o que é limitado pelas condições ambientais de cada localidade. O aumento do coeficiente de transferência de calor do fluído operante, pode ser uma das soluções mais viáveis para a otimização dos equipamentos de troca de calor, por meio da substituição dos fluídos térmicos convencionais por nanofluídos, esperando-se melhora na eficiência dos equipamentos. São inúmeras as possíveis aplicações para as quais os nanofluídos podem ser utilizados, dentre elas o aquecimento solar de água (FERNANDES, 2013).

Muhammad et al. (2016) fizeram uma revisão de estudos sobre o desempenho térmico de coletores solares térmicos de placa plana, que obtiveram ganhos de eficiência próximos de $20 \%$ e potencial de redução da área de coletores de até $25 \%$, quando utilizados nanofluídos obtidos a partir de nanopatículas de diferentes materiais em diferentes percentuais de diluição, na comparação da eficiência ao se utilizar água como fluído de troca de calor. Sharafeldin e Gróf (2018), também identificaram que um coletor solar pode se tornar-se mais econômico, obtendo uma redução da área de superfície em cerca de $20 \%$ usando nanofluídos em vez de água como fluído convencional. Tecnicamente, a integração do sistema de aquecimento solar a um processo produtivo industrial tem certo grau de complexidade e exige disponibilidade de uma área adequada para a instalação dos painéis solares. Estudos de Mekhilef et al. (2011), bem como de Quijera et al. (2011) e de Kulkarni et al. (2008) mencionam a importância do espaço disponível e efetivamente ocupado pelo sistema de coletores e armazenamento na área da fábrica para analisar a viabilidade do sistema. 
O objetivo central deste estudo é realizar uma revisão de artigos referentes ao estágio atual de aplicação de nanofluídos em sistemas de aquecimento solar, identificando possibilidades de aumentar a eficiência desses sistemas, buscando a redução da área total necessária de coletores, o que consequentemente resultará na redução do espaço ocupado pelo sistema de aquecimento e na redução dos custos para a instalação dos mesmos.

\section{REVISÃO DA LITERATURA}

\section{Nanofluídos}

Conforme Muhammad et al. (2016), os nanofluídos são um tipo avançado de fluído contendo uma pequena quantidade de nanopartículas (geralmente menores do que $100 \mathrm{~nm}$ ) uniforme e estavelmente suspensas no líquido. Recentemente, nanofluídos têm sido testados como fluídos de transferência de calor para melhorar o desempenho dos dispositivos coletores solares.

Para Matias (2016), os nanofluídos consistem na dispersão de nanomateriais, como, por exemplo, nanopartículas, nanotubos, nanofibras, nanofios, nanofolhas (2D), nanocilindros (3D) ou partículas nanométricas em forma de gotas (nanodroplets), em fluídos base, como por exemplo etileno-glicol (EG), água, óleos ou até mesmo líquidos iônicos. Após dispersão uniforme formam uma mistura estável e com propriedades diferentes das dos componentes isolados, ou seja, possuem melhores propriedades termo físicas como condutividade térmica, difusividade térmica, viscosidade e coeficientes de transferência de calor por convecção, quando comparados com os respectivos fluídos base. Existem inúmeros tipos de nanomateriais utilizados na preparação de nanofluídos, tais como, materiais metálicos (prata, cobre, alumínio, ferro, níquel, paládio, entre outros) e materiais não metálicos (silício, alumina, grafite, nanotubos de carbono, etc.). Os nanofluídos podem ser preparados por dois métodos:

- Método two-step, o mais utilizado, no qual os nanomateriais são previamente transformados em pó por métodos físicos ou químicos, onde as partículas apresentam tamanho nanométrico e depois são dispersos num fluído base, utilizando agitação por ultrassons, agitação de força magnética, dispersão por pressurização, homogeneização ou moinho de bolas. Este é o método mais econômico de preparação, mas neste método as nanopartículas têm tendência à aglomeração, podendo ser evitada com a adição de surfactantes com o objetivo de estabilizar os nanofluídos.

- $\quad$ Método one-step, cujo intuito é reduzir a aglomeração das nanopartículas, que consiste em produzir e dispersar as nanopartículas no fluído base ao mesmo tempo, eliminando passos como secagem, armazenamento, transporte e dispersão de nanopartículas, reduzindo a aglomeração e aumentando a estabilidade do nanofluído. Este método é bastante eficiente em dispersar uniformemente as nanopartículas, no entanto apresenta elevados custos de produção em escala industrial.

Leong (2016) menciona que na maioria das pesquisas relacionadas a coletores solares os nanofluídos foram produzidos por meio do método de duas etapas.

Para Fernandes (2013) o estudo dos nanofluídos na transferência de calor requer conhecimento prévio sobre o melhor método de produção e sobre a estabilidade coloidal do nanofluído para garantir o bom funcionamento dos novos fluídos térmicos, além de requerer conhecimento aprofundado das propriedades térmicas necessárias ao estudo da transferência de calor, como a condutividade térmica, o calor específico e a viscosidade. Este autor detalha as propriedades dos nanofluídos a seguir:

- Estabilidade: mantém as nanopartículas homogeneamente diluídas no fluído base, mantendo os requisitos de aglomeração reduzida, pouca sedimentação e integridade estrutural para as condições de estudo, o que pode ser obtido pela utilização de ultrassons na preparação de nanofluídos, o controle do PH das nanopartículas e da adição de dispersantes (surfactantes) para evitar sedimentação. Na revisão de trabalhos de outros autores, ele encontrou tempos de estabilidade de nanofluídos de 10 dias, 1 mês, 2 meses, 3 meses e 2 anos, ou seja, resultados muito diferentes entre si. Em seu próprio estudo, Fernandes (2013) previu um tempo de vida útil de aproximadamente 4 anos, para nanofluídos com nanotubos de carbono.

Densidade: é a relação massa por unidade de volume de uma determinada substância, cujo aumento excessivo nos nanofluídos aplicados a sistemas térmicos influencia fortemente a capacidade e eficiência de bombeamento em coletores de convecção forçada. A densidade de nanofluídos varia tendencialmente para um valor superior com a concentração de nanopartículas, e varia inversamente com a temperatura. 
- Calor específico: é uma grandeza física que define a variação térmica de uma determinada substância ao receber determinada quantidade de calor, o que permite saber a quantidade de energia que um corpo consegue transmitir a outro corpo. O calor específico de um nanofluído depende do calor específico do fluído base e do das nanopartículas, da concentração em volume de nanopartículas e da temperatura. A literatura sugere que o calor específico do nanofluído diminui com o aumento da concentração de volume e aumenta com a temperatura.

- $\quad$ Viscosidade: é propriedade crucial no dimensionamento de sistemas com convecção forçada. Um aumento da condutividade térmica é diretamente relacionando com a viscosidade. A análise realizada pelo autor mostra que a viscosidade aumenta com a adição de nanopartículas ao fluído base, em função do aumento da concentração de nanopartículas, havendo também uma diminuição da viscosidade com o aumento da temperatura.

- $\quad$ Condutividade térmica: nos nanofluídos depende de diversos fatores, sendo que ela aumenta linearmente com a concentração volumétrica de nanopartículas bem como com a redução do tamanho médio das nanopartículas.

Tabela 1 - Condutividade térmica de nanofluídos

\begin{tabular}{|c|c|c|}
\hline Nanofluído & $\begin{array}{l}\text { Concentração } \\
\text { Volumétrica }(\%)\end{array}$ & $\begin{array}{l}\text { Aumento de } \\
\text { Condutividade } \\
(\%)\end{array}$ \\
\hline $\mathrm{Cu} / \mathrm{EG}$ & 0,3 & 0,50 \\
\hline SiO2/água & 1 & 3,23 \\
\hline SnO2/água & 0,024 & 7 \\
\hline $\mathrm{Cu} / \mathrm{EG}$ & 5 & 8 \\
\hline MWCNT/água & 3 & 13 \\
\hline $\mathrm{Cu} / \mathrm{EG}$ & 11 & 16 \\
\hline ZnO/EG-água & 10 & 17 \\
\hline MWCNT/EG & 1,5 & 17 \\
\hline SiO2/água & 4 & 23 \\
\hline CuO/EG-água & 10 & 32 \\
\hline $\mathrm{Fe} 3 \mathrm{O} 4 / q u e r o s e n e$ & 1 & 34 \\
\hline Al2O3/EG-água & 10 & 35 \\
\hline $\mathrm{Cu} / \mathrm{EG}$ & 33 & 46 \\
\hline Au/água & 0,00026 & 48 \\
\hline $\mathrm{Al}-\mathrm{Cu} / \mathrm{EG}$ & 1,5 & 200 \\
\hline
\end{tabular}

Fonte: Adaptado de Fernandes, 2013

Na Tabela 1 podem ser vistos diferentes variações da condutividade térmica de diferentes nanofluídos com diferentes concentrações volumétricas, encontradas pelo autor em diferentes estudos de pesquisa.

- $\quad$ Coeficiente de transferência de calor por convecção (h): este autor mostra que este coeficiente aumenta nos nanofluídos em comparação com os fluídos base, que a geometria da nanopartícula influencia no coeficiente, sendo maior quando são utilizados nanotubos, bem como também é maior em nanopartículas de menor tamanho.

Tabela 2 - Coeficientes empíricos de transferência de calor por convecção

\begin{tabular}{lccc}
\hline Nanofluído & $\begin{array}{l}\text { Concentração } \\
\text { volumétrica }(\boldsymbol{\%})\end{array}$ & Escoamento & Aumento de h (\%) \\
\hline ZrO2/água & 1,32 & Laminar & 3 \\
\hline Al2O3/água & 0,3 & Laminar & 8 \\
\hline TNT/água & 2,5 & Laminar & 13,5 \\
\hline Al2O3-Cu/água & 0,1 & Laminar & 13,56 \\
\hline
\end{tabular}




\begin{tabular}{lclc}
\hline TiO2/água & 1 & Turbulento & 14 \\
\hline Grafite/água & 2 & Laminar & 22 \\
\hline MWCNT/água & 0,12 & Laminar & 25 \\
\hline TiO2/água & 2 & Turbulento & 26 \\
\hline Al2O3/água & 6 & Laminar & 27 \\
\hline CuO/EG-água & 6 & Turbulento & 35 \\
\hline Al2O3/água & 1,6 & Laminar & 41 \\
\hline MWCNT/água & 0,5 & Turbulento & 47 \\
\hline Cu/água & 2 & Laminar & 60 \\
\hline Cu/água & 2 & Turbulento & 60 \\
\hline MWCNT/água & 0,45 & Turbulento & 159,3 \\
\hline
\end{tabular}

Fonte: Adaptado de Fernandes, 2013

Na Tabela 2 podem ser vistos valores do aumento percentual do coeficiente de transferência de calor por convecção encontrados pelo autor em diferentes estudos, para diferentes tipos de nanofluídos, com diferentes concentrações volumétricas e com diferentes velocidades de escoamento. Verifica-se maior aumento do coeficiente nos nanofluídos com maior concentração de partículas e para regimes turbulentos de escoamento.

Trabalhos pesquisados pelo autor verificaram que as correlações clássicas não conseguem prever a transferência de calor para os nanofluídos. Não há consenso para a determinação do número de Nusselt, embora seja possível afirmar que nanofluídos têm maior número de Prandtl do que o fluido base, diminuindo com o aumento da temperatura.

\section{Nanofluídos aplicados ao aquecimento solar de água}

A seguir são apresentados resultados obtidos em diversos experimentos de coletores solares com a utilização de nanofluídos:

Mahian et al. (2013) revisaram aplicações de nanofluídos em sistemas solares térmicos, concluindo que a eficiência aumenta para sistemas de circulação forçada, podendo resultar em economia de eletricidade e combustível. Apontaram como principais desafios: à redução do custo dos nanofluídos devido às dificuldades de produção, superar a questão da aglomeração das nanopartículas em altas temperaturas, o aumento da viscosidade do nanofluído que leva aos aumentos da queda de pressão e da potência necessária ao bombeamento e erosão causada pelas nanopartículas aos sistemas.

Sarsam et al. (2015) revisou investigações do desempenho de coletores solares de placa plana usando nanofluídos como fluídos de trabalho, a maioria concentrada no uso de nanofluídos de Al2O3 e MWCNT, e alguns outros à base de $\mathrm{Ag}, \mathrm{TiO} 2, \mathrm{ZnO}, \mathrm{Fe} 2 \mathrm{O} 3, \mathrm{CuO}, \mathrm{SiO} 2$ e SWCNT. As maiores eficiências foram obtidas utilizando nanofluídos baseados em nanoestruturas de carbono em baixas concentrações das nanopartículas, concluindo-se que as nanopartículas à base de carbono são o tipo mais promissor de nanopartículas dispersas em água em concentrações muito baixas, uma vez que apresentaram maior estabilidade de dispersão, menor custo, com pequenos aumentos, tanto de viscosidade, quanto de queda de pressão e da potência de bombeamento. Nas suas investigações não encontrou nenhuma pesquisa experimental que tenha abordado o efeito do uso de surfactantes em temperaturas superiores a $60^{\circ} \mathrm{C}$, em que ocorre o dano da ligação entre o surfactante e as nanopartícula, resultando na perda de estabilidade e no assentamento das nanopartículas. Os desafios mais significativos no uso de nanofluídos em coletores solares de placas planas podem ser resumidos como o alto custo das nanopartículas, a instabilidade das nanopartículas e o aumento da viscosidade, resultando no aumento da queda de pressão e da potência de bombeamento.

Kasaeian et al. (2015) mencionaram o aumento da eficiência para o coletor solar de tubo evacuado utilizando nanofluídos CuO/água, obtendo um aumento de $30 \%$ para o coeficiente de transferência de calor por evaporação e também apontaram como maior desafio reduzir o custo e desenvolver a produção de nanopartículas.

Beicker (2016) estudou o comportamento de nanofluídos de ouro e nanotubos de carbono de paredes múltiplas dispersos em água destilada utilizando coletores solares de absorção direta, sem concentração, construídos pela autora. Em relação à estabilidade, após cinco dias de teste os nanofluídos de ouro apresentaram 
indícios de decantação, enquanto os de nanotubos de carbono permaneceram estáveis. Os resultados da energia total armazenada no período de aquecimento, taxa de variação da temperatura, taxa de absorções específica e a razão de energia armazenada confirmaram dados da literatura que afirmam existir para os nanofluídos uma concentração volumétrica considerada "ótima", acima da qual se torna indiferente ou inviável a adição de nanopartículas, sendo que a concentração volumétrica ideal para aplicações estudadas foi de 0,004\% para as nanopartículas de ouro e $0,001 \%$ para os nanotubos de carbono. Os testes indicaram que com o alto custo de produção de nanopartículas de ouro e a visível degradação das amostras após exposição prolongada à radiação solar, a dispersão de nanotubos de carbono em água seria mais viável para aplicações de conversão fototérmica.

Muhammad et al. (2016) realizaram uma revisão sobre aplicações de nanofluídos em coletores solares de tubo evacuado, encontrando como resultados aumentos de eficiência do coletor de 16,75\% e 42,5\% utilizando nanofluído de óxido de titânio a base de água e de 71,84\% com nanotubos de carbono, em relação à operação com apenas água. Estudos com nanotubos de carbono em coletor solar de tubo em "U" apresentaram um aumento de eficiência de 62,8\%, valor maior ao encontrado com nanofluídos a base de Al2O3, $\mathrm{CuO}, \mathrm{SiO} 2$ e $\mathrm{TiO} 2$. Outro estudo utilizando nanofluído $\mathrm{CuO}$ /água em coletor de tubo evacuado apresentou desempenho térmico aumentado em cerca de $30 \%$ em comparação com o da água deionizada. O autor alertou também que os principais desafios dos nanofluídos para dispositivos solares são principalmente o alto custo do nanofluído devido a limitações de produção, a instabilidade e a aglomeração de nanopartículas, bem como energia de bombeamento e queda de pressão.

Leong (2016) também confirma que os principais desafios a serem superados na aplicação de nanofluídos em coletores solares são: a perda de estabilidade dos nanofluídos no decorrer do tempo (problema que permanece sem solução), o que impede a comercialização de nanofluídos; o aumento da viscosidade pela adição de nanopartículas no fluído base, o que resulta na necessidade de maior potência de bombeamento para operar o coletor, aumentando o custo de operação do sistema. No entanto este último item pode não ser um problema se a economia de energia devido à melhoria da eficiência do coletor solar for muito maior do que o custo de operação, o que deve ser levado em conta em uma análise técnico-econômica.

Elsheikh et al. (2017) identificaram avanços nas aplicações de nanofluídos em sistemas de energia solar nos quais as propriedades termo físicas superiores dos nanofluídos resultam em um aprimoramento significativo no processo de transferência de calor, que por sua vez resulta na redução do tamanho dos dispositivos solares. Entretanto, também identificaram como desafios a serem superados evitar a degradação de surfactantes e a aglomeração de nanopartículas para aplicações de alta temperatura e a redução do custo de produção dos nanofluídos. Problemas identificados foram a divergência nos resultados obtidos por diferentes pesquisadores e a falta de estudos aplicados a casos reais.

Iranmanesh et al. (2017) investigaram experimentalmente o efeito de nanofluídos de grafeno em fluído base água destilada no desempenho térmico de um coletor solar de tubo evacuado resultando que a eficiência térmica do coletor solar aumentou com o uso de nanofluídos de grafeno com diferentes concentrações. A eficiência térmica do sistema foi aumentada para 90,7\% tendo ficado 35,8\% acima da eficiência da água destilada. Os resultados indicaram que, aumentando a porcentagem de massa das nanopartículas, o ganho de energia térmica também aumenta, atingindo uma temperatura mais alta do fluído na saída dos coletores quando nanosheets (folhas) de grafeno são usados.

Verma et al. (2017) analisaram uma ampla variedade de nanofluídos em coletores solares de placa plana. Os resultados do experimento apontaram um aumento na eficiência exergética e consequentemente energética dos nanofluídos com nanotubos de carbono de $29,32 \%$ e $23,47 \%$, respectivamente, em comparação com a água do fluído base. O aumento na eficiência exergética de outros nanofluídos foi de 21,46\% em grafeno, 16,67\% em cobre, 10,86\% em alumínio, 6,97\% em titânio e 5,74\% em nanofluídos a base de óxido de silício. O incremento na eficiência energética dos coletores foi de 16,93\% para grafeno, 12,64\% para cobre, 8,28\% para alumínio, 5,09\% para titânio e 4,08\% para óxido de silício. Um coletor solar pode se tornar uma opção econômica, tornando-o mais compacto, reduzindo a área de superfície em cerca de $20 \%$ usando nanofluídos com nanotubos de carbono/água em vez de água fluida convencional. Os autores também destacam desafios a serem superados, quanto ao custo, produção em massa, estabilidade e mecânica do comportamento das nanopartículas no fluído base.

Zeiny et al. (2018), realizaram um experimento em um simulador solar de alta qualidade a partir do conceito de absorção direta, utilizando nanofluídos de ouro, cobre, de negro de fumo e seus híbridos, para avaliar seu desempenho em termos de eficiência de conversão fototérmica, taxa de absorção específica e custo de materiais. $\mathrm{O}$ estudo revelou que embora os nanofluídos de ouro tenham uma taxa de absorção específica elevada, o seu custo 
dispendioso limita a sua utilização prática, enquanto os nanofluídos de negro de fumo são economicamente mais viáveis.

Raj e Subudhi (2018) realizaram uma revisão de vários trabalhos de pesquisa realizados sobre a aplicação de nanofluídos em dispositivos solares térmicos, tendo constatado que uso de nanofluido no lugar do fluído base melhorou o efeito nas propriedades termo físicas, como a condutividade térmica em coletores solares de placa plana. Desafios e dificuldades no uso de nanofluídos: alto custo de nanopartículas é a principal limitação na utilização de nanofluídos, o método de preparação do nanofluido é dispendioso e moroso, nanofluido não é estável por um longo período de tempo, através da adição de nanopartículas no fluído base há um aumento na viscosidade devido ao qual há aumento da queda de pressão sendo necessária maior potência de bombeamento, o uso contínuo a longo prazo do nanofluido pode causar erosão das paredes na tubulação dos coletores. As conclusões mais importantes indicam que a dispersão adequada de nanopartículas e estabilidade a longo prazo são necessárias para aumentar a eficiência do coletor solar, a eficiência do coletor aumenta com o aumento da fração volumétrica mas com o aumento adicional a eficiência diminui porque as forças viscosas aumentam e a taxa de transferência de calor diminui, a maioria das pesquisas centrou-se na placa plana e coletor solar de absorção direta.

Mahbubul et al. (2018) realizaram um estudo para analisar o efeito do nanotubo de carbono de parede única no desempenho de um sistema de coletores solares de tubos evacuados, inicialmente operado com água. O sistema de 14 coletores ocupou uma área de $116 \mathrm{~m}^{2}$, com área efetiva de $42 \mathrm{~m}^{2}$, com refletores embutidos na parte inferior de cada unidade de coletor solar para ganhar mais calor. Os resultados indicaram eficiência 56,7\% quando o sistema é operado com água e $66 \%$ quando operado com nanofluído. Na saída do sistema a temperatura máxima foi de $120,6^{\circ} \mathrm{C}$. Dentre os demais autores citados anteriormente neste trabalho, este foi o único sistema dessa dimensão encontrado, pois se tratava de um sistema real instalado, sendo que os demais utilizaram sistemas experimentais reduzidos.

Sharafeldin e Grof (2018) realizaram um estudo da utilização de nanofluído CeO2 e fluído base água em coletor solar de tubo evacuado. O nanofluído utilizado foi produzido pelos autores. O trabalho conclui que o aumento máximo da diferença de temperatura entre a saída e a entrada do sistema é de 37,3\% no uso de nanofluído CeO2 se comparada à água no mesmo fluxo de massa. O ganho de calor útil aumentou com o uso de nanofluídos. Além disso, quanto mais nanopartículas de $\mathrm{CeO} 2$ adicionadas à água, mais calor foi absorvido. Com base nisso, a elevação máxima do ganho de calor obtida no experimento foi maior em 42,3\% do que o ganho de calor no caso da água pura. O aumento máximo da taxa de transferência de encontrado calor foi de 34,66\% em comparação com a água na mesma taxa de fluxo de massa.

\section{METODOLOGIA}

Com o objetivo de se identificar na literatura atual o estágio de desenvolvimento da utilização de nanofluídos em sistemas de aquecimento solar de água, foi realizado um trabalho de revisão bibliográfica. Os artigos analisados foram encontrados através da busca pelos termos "nanofluídos", "aquecimento solar" e "aquecimento de água", na base ScienceDirect (https://www.sciencedirect.com/). A análise dos mesmos buscou identificar o estágio atual do desenvolvimento de pesquisas e da aplicação das possibilidades de aumentar a eficiência dos fluídos de troca de calor em coletores solares, a fim de poder proporcionar a redução da área total de coletores necessária às aplicações de aquecimento solar de água, reduzindo a necessidade de espaço bem como os custos para a instalação de sistemas solares de aquecimento de água, como resultado da utilização de nanofluídos. Nesse trabalho é apresentada a contribuição dos artigos selecionados, em ordem cronológica de suas publicações, sempre que possível, para que se possa identificar as vantagens e limitações do uso de nanofluídos como fluídos de trabalho em sistemas de aquecimento solar de água, os tópicos comuns discutidos pelos autores consultados e por fim, os estudos que ainda precisam ser realizados para superar os desafios das limitações atuais ao uso comercial de nanofluídos.

\section{RESULTADOS}

Coletores solares de placa plana são os tipos mais amplamente utilizados e conhecidos, tendo baixa eficiência em relação a outros tipos de coletores, aquecem o fluído apenas em baixas temperaturas, ou seja, entre 
$60^{\circ} \mathrm{C}$ e $80^{\circ} \mathrm{C}$ e têm como fluído de trabalho água. Coletores solares de tubo evacuado são mais eficientes do que os de placa plana, possibilitando aquecer o fluído até cerca de $120^{\circ} \mathrm{C}$ e $150^{\circ} \mathrm{C}$, tendo por fluído de trabalho também água. A aplicação de coletores solares para aquecimento tem limitações de eficiência, o que exige grandes áreas de coletor para atender grandes demandas de água quente, por exemplo, nas indústrias.

A revisão da literatura mostra que nanofluídos poderiam substituir fluídos convencionais nos coletores solares, e, devido ao aumento da condutividade térmica do fluído, pderiam aumentar a eficiência térmica dos coletores, resultando em um aumento da temperatura final do fluído ou redução da área dos coletores. Estudos recentes mostram que com nanofluídos poderia haver ganhos acima de $20 \%$ ou $30 \%$ na eficiência dos coletores em comparação à utilização de água como fluído de trabalho. Foi possível identificar que apesar de os autores terem realizado experimentos com nanopartículas de diferentes materiais, aqueles que apresentaram a melhor relação de resultados e menores custos foram as nanopartículas de carbono, principalmente nanotubos e carbono e grafeno. Há que se observar que, exceto para um dos estudos relatados, os experimentos foram realizados em coletores solares de pequena dimensão ou em simuladores solares em laboratórios e que as nanopartículas foram produzidas pelos próprios autores dos estudos, bem como há diferença de procedimentos entre os diferentes experimentos investigados, o que pode gerar certa inconsistência na comparação dos dados obtidos por diferentes pesquisadores.

Entretanto, ainda existem vários e grandes desafios a serem superados para a utilização de nanofluídos em escala industrial:

A produção de nanofluídos ainda depende de processos e equipamentos relativamente complexos e especializados, o que dificulta a sua produção em escala industrial.

Quanto à vida útil do nanofluído, não é possível uma previsão muito precisa, uma vez que foram poucos os estudos realizados nesse sentido e não há consenso quanto à vida útil dos nanofluídos testados. Com o passar do tempo e principalmente devido a temperaturas elevadas, as nanopartículas tendem a se aglomerar ou a decantar, alterando as características do nanofluído homogêneo, sobretudo a estabilidade. A vida útil está diretamente relacionada à frequência da necessidade de troca do fluído do sistema térmico, representando gastos adicionais à operação.

Nanofluídos apresentam viscosidade maior do que o fluído base, o que resulta em perda de pressão no escoamento e na necessidade de maior potência de bombeamento, resultando em um aumento do custo de operação do sistema, que só será compensado se o aumento de eficiência térmica representa um ganho superior em economia. Além disso, no longo prazo as nanopartículas podem causar erosão das paredes da tubulação dos coletores.

\section{CONSIDERAÇÕES FINAIS}

Coletores solares de placa plana são um dos tipos mais amplamente utilizados e conhecidos, tendo eficiência limitada e aquece o fluído apenas em baixas temperaturas, ou seja, entre $60^{\circ} \mathrm{C} \mathrm{e} 80^{\circ} \mathrm{C}$, tendo por fluído base água. Coletores solares de tubo evacuado são mais eficientes do que coletores de placa plana, possibilitando aquecer o fluído até cerca de $120^{\circ} \mathrm{C}$ e $150^{\circ} \mathrm{C}$, tendo por fluido base água. A aplicação de coletores solares para aquecimento tem limitações de eficiência, o que exige grandes áreas coletoras para sistemas que devam atender grandes demandas de água quente, por exemplo, nas indústrias.

Nanofluídos são dispersões de nanopartículas de diferentes materiais que podem substituir fluidos convencionais nos coletores solares, e consequentemente aumentar a condutividade térmica dos fluídos de trabalho, bem como aumentar a eficiência térmica dos coletores, resultando em aumento da temperatura final do fluído ou redução da área dos coletores. Apesar de os autores terem realizado experimentos com nanopartículas de diferentes materiais, aqueles que apresentaram a melhor relação de resultados e menores custos foram as nanopartículas de carbono, principalmente nanotubos e carbono e grafeno.

Pode-se concluir que os nanofluídos representem uma grande oportunidade de melhoria de operação dos sistemas de aquecimento solar, podendo proporcionar aumentos de eficiência térmica desses sistemas em até $25 \%$ ou redução da área coletora em até $20 \%$. A redução da área coletora de um sistema de aquecimento solar é uma vantagem significativa, pois além e proporcionar a redução o custo de investimento para a implantação do sistema, pode significar também a redução da necessidade de espaço para a montagem do sistema, o que vem a ser outra vantagem, uma vez que nem sempre há muito espaço ocioso disponível no local da instalação.

Entretanto, a utilização de nanofluídos como fluído de trabalho em coletores solares de placa plana e de tubos evacuados ainda não é viável para aplicações reais, pois os nanofluídos ainda não são produzidos em escala comercial, apresentam alto custo de obtenção, têm problemas de estabilidade, principalmente em temperaturas mais 
elevadas, não se pode prever com segurança o tempo de vida útil dos mesmos e ainda devido ao aumento da viscosidade do fluído térmico provocado pelas nanopartículas, o custo de bombeamento para a circulação do fluído aumenta, diminuindo a viabilidade dos sistemas, além de poder provocar desgastes internos aos tubos dos coletores.

Este estudo pode contribuir para a utilização da energia solar térmica, apresentando as vantagens da utilização de nanofluídos em coletores solares, com vistas a melhorar o desempenho dos mesmos, resultando na redução da área de coletores necessária aos sistemas de aquecimento solar e, consequentemente, na redução do custo de implantação dos mesmos, apesar de que certos desafios ainda tenham que ser vencidos, tanto em aspectos técnicos como econômicos, para que seja possível a produção de nanofluídos em escala comercial, o que requer o desenvolvimento de novos estudos neste campo.

\section{REFERÊNCIAS}

BEICKER, Carolina Lau Lins. Análise experimental da conversão fototérmica em nanofluídos de ouro e nanotubos de carbono em água. 2016. Dissertação de Mestrado. Universidade Federal de Uberlândia.

ELSHEIKH, A. H. et al. Applications of nanofluids in solar energy: A review of recent advances. Renewable and Sustainable Energy Reviews, 2017.

FERNANDES, Fábio Alexandre Teixeira. Caracterização da transferência de calor em nanofluídos. 2013. Dissertação de Mestrado. Universidade de Aveiro.

IRANMANESH, Soudeh et al. Thermal performance enhancement of an evacuated tube solar collector using graphene nanoplatelets nanofluid. Journal of cleaner production, v. 162, p. 121-129, 2017.

KASAEIAN, Alibakhsh; ESHGHI, Amin Toghi; SAMETI, Mohammad. A review on the applications of nanofluids in solar energy systems. Renewable and Sustainable Energy Reviews, v. 43, p. 584-598, 2015.

KULKARNI, Govind N.; KEDARE, Shireesh B.; BANDYOPADHYAY, Santanu. Design of solar thermal systems utilizing pressurized hot water storage for industrial applications. Solar Energy, v. 82, n. 8, p. 686-699, 2008.

LEONG, K. Y. et al. An overview on current application of nanofluids in solar thermal collector and its challenges. Renewable and Sustainable Energy Reviews, v. 53, p. 1092-1105, 2016. 
MAHBUBUL, I. M. et al. Carbon nanotube nanofluid in enhancing the efficiency of evacuated tube solar collector. Renewable Energy, 2018.

MAHIAN, Omid et al. A review of the applications of nanofluids in solar energy. International Journal of Heat and Mass Transfer, v. 57, n. 2, p. 582-594, 2013.

MATIAS, Inês Andreia Simão. Nanofluídos para aplicações energéticas. 2016. Tese de Doutorado. Instituto Superior de Engenharia de Lisboa.

MEKHILEF, Saidur; SAIDUR, Rahman; SAFARI, Azadeh. A review on solar energy use in industries. Renewable and Sustainable Energy Reviews, v. 15, n. 4, p. 1777-1790, 2011.

MUHAMMAD, Mahmud Jamil et al. The use of nanofluids for enhancing the thermal performance of stationary solar collectors: a review. Renewable and Sustainable Energy Reviews, v. 63, p. 226-236, 2016.

MUHAMMAD, Mahmud Jamil et al. Thermal performance enhancement of flat-plate and evacuated tube solar collectors using nanofluid: a review. International Communications in Heat and Mass Transfer, v. 76, p. 6-15, 2016.

QUIJERA, José Antonio; ALRIOLS, María González; LABIDI, Jalel. Integration of a solar thermal system in a dairy process. Renewable Energy, v. 36, n. 6, p. 1843-1853, 2011.

RAJ, Pankaj; SUBUDHI, Sudhakar. A review of studies using nanofluids in flat-plate and direct absorption solar collectors. Renewable and Sustainable Energy Reviews, v. 84, p. 54-74, 2018. 